\title{
HEAT KERNEL ANALYSIS ON INFINITE DIMENSIONAL GROUPS
}

\author{
MARIA GORDINA
}

\begin{abstract}
The paper gives an overview of our previous results concerning heat kernel measures in infinite dimensions. We give a history of the subject first, and then describe the construction of heat kernel measure for a class of infinite-dimensional groups. The main tool we use is the theory of stochastic differential equations in infinite dimensions. We provide examples of groups to which our results can be applied. The case of finite-dimensional matrix groups is included as a particular case.
\end{abstract}

\section{Motivation AND HISTORY.}

In this paper we review our results in [7], [8], [9], [11] and show how they fit into a broader picture. In order to see the challenges this study presents, we first review what is known in finite dimensions and for the flat infinite-dimensional case. Our main results concern analogues of heat kernel (Gaussian) measures on infinitedimensional groups.

1.1. Finite-dimensional noncommutative case. The initial motivation for our work was the following result for finite-dimensional Lie groups. It has a long history which we address later in this section. Let $G$ be a finite-dimensional connected complex Lie group with a Lie algebra $\mathfrak{g}$. The Lie algebra is a complex Hilbert space with a norm $|\cdot|$, and we denote by $\left\{\xi_{i}\right\}_{i=1}^{2 n}$ an orthonormal basis of $\mathfrak{g}$ as a real vector space. By identifying $\mathfrak{g}$ with left-invariant vector fields at the identity $e$ we can define the derivative

$$
\partial_{i} f(g)=\left.\frac{d}{d t}\right|_{t=0} f\left(g e^{t \xi_{i}}\right), g \in G
$$

and the Laplacian

$$
\Delta f=\sum \partial_{i}^{2} f
$$

The heat kernel measure $\mu_{t}$ has the heat kernel as its density with respect to a Haar measure $d x$. The heat kernel is the fundamental solution to the heat equation

$$
\begin{gathered}
\frac{\partial \mu_{t}(x)}{\partial t}=\frac{1}{4} \Delta \mu_{t}(x), t>0, x \in G, \\
\mu_{t}(x) d x \stackrel{\text { weakly }}{\underset{t \rightarrow 0}{\longrightarrow}} \delta_{e}(d x) .
\end{gathered}
$$

\footnotetext{
${ }^{1}$ Research supported by the NSF Grant DMS-0306468.

Date: December 14, 2004.

Key words and phrases. Heat kernel analysis, infinite-dimensional Lie groups and Lie algebras, von Neumann algebras, noncommutative $L^{p}$-space, Fock space.
} 
We can define holomorphic functions on $G$ as functions whose derivatives are complex linear. The $k$ th derivative $D^{k} f(e)$ is defined as the tensor

$$
\left\langle D^{k} f(g), \xi_{i_{1}} \otimes \ldots \otimes \xi_{i_{k}}\right\rangle=\left(\partial_{i_{1}} \ldots \partial_{i_{k}} f\right)(g) .
$$

Denote by $\mathcal{H} L^{2}\left(G, \mu_{t}\right)$ the space of holomorphic functions which are squareintegrable with respect to $\mu_{t}$. It is known to be a closed subspace. The following identity represents an isometry between $\mathcal{H} L^{2}\left(G, \mu_{t}\right)$ and a certain space of tensors over the Lie algebra $\mathfrak{g}$.

$$
\|f\|_{\mathcal{H} L^{2}}^{2}=\int_{G}|f(z)|^{2} d \mu_{t}(z)=\sum_{k=0}^{\infty} \frac{t^{k}}{k !}\left|D^{k} f(e)\right|^{2} .
$$

This identity was first proved by B. Driver, L. Gross for connected complex Lie groups in [5]. In particular, if $G=\mathbb{C}^{n}$, then the tensors $D^{k} f$ are symmetric, and with this norm they form a bosonic Fock space (see V. Bargmann, I. Segal in [1], $[2],[21])$.

Our goal is to describe similar results for some infinite-dimensional groups. As we will see later, the approach described above is not applicable in that situation, but we will make use of the following probabilistic approach. Assume that $G$ is a matrix group. Then we can make sense of multiplying elements from $G$ and $\mathfrak{g}$. Instead of using the heat equation to define the heat kernel measure, we can view this measure $\mu_{t}$ as the transition probability of the Wiener process $X_{t}$ on $G$ satisfying the stochastic differential equation

$$
d X_{t}=X_{t} d W_{t}, X_{0}=e,
$$

where $W_{t}=\sum_{i=1}^{2 n} W_{t}^{i} \xi_{i}$ is the Wiener process on $\mathfrak{g}$ and $W_{t}^{i}$ are independent onedimensional Wiener processes.

1.2. Finite-dimensional commutative case. The identity in Equation 1.1 has a long history. It appeared in the commutative setting $\left(G=\mathbb{C}^{n}\right)$ as follows. Consider a system of $n$ classical harmonic oscillators. They satisfy Newton's equation in $\mathbb{R}^{n}$

$$
m_{j} x_{j}^{\prime \prime}=-k_{j} x_{j}, \quad j=1, \ldots n .
$$

The corresponding quantum state space for the system is $L^{2}\left(\mathbb{R}^{n}, d x\right)$ with the Hamiltonian

$$
\mathfrak{H}=-\frac{1}{2} \Delta+\frac{1}{8 t^{2}}|x|^{2} .
$$

Then the ground state for this Hamiltonian is

$$
\varphi=\frac{e^{-|x|^{2} / 4 t}}{(2 \pi t)^{n / 4}}, \mathfrak{H} \varphi=\frac{n}{4 t} \varphi,
$$

and the heat kernel $\rho_{t}$ satisfies

$$
\varphi^{2}=\rho_{t}=\frac{e^{-|x|^{2} / 2 t}}{(2 \pi t)^{n / 2}} .
$$


This works if $n<\infty$, but not in infinite dimensions where there is no analogue of the Lebesgue measure. However, if the Lebesgue measure is replaced by the Gaussian measure, we can extend the setting to infinite dimensions. Let us first explain how it can be done in the finite dimensional commutative case.

Let $d \rho_{t}=\rho_{t} d x, d \mu_{t}=\frac{e^{-|z|^{2} / t}}{(\pi t)^{n}} d z$ be the Gaussian measures respectively on $\mathbb{R}^{n}$ and $\mathbb{C}^{n}$. By $\mathfrak{F}_{s}=\oplus_{m=0}^{\infty} H^{\otimes_{s} m}$ denote a bosonic Fock space, where $H=\mathbb{C}^{n}$ is a state space of a single particle. As before $\mathcal{H} L^{2}\left(\mathbb{C}^{n}, \mu_{t}\right)$ is the space of square integrable holomorphic functions. In this situation we can describe the isometries between the three spaces as follows. Let $H_{k}(x)$ be an Hermite polynomial, then $H_{k_{1}}\left(x_{j_{1}}\right) \cdot \ldots \cdot H_{k_{n}}\left(x_{j_{n}}\right)$ form an orthonormal basis of $L^{2}\left(\mathbb{R}^{n}, \rho_{t}\right)$, and thus

$$
\begin{aligned}
B\left(H_{k_{1}}\left(x_{j_{1}}\right) \cdot \ldots \cdot H_{k_{n}}\left(x_{j_{n}}\right)\right) & =x_{j_{1}}^{k_{1}} \otimes_{s} \ldots \otimes_{s} x_{j_{n}}^{k_{n}} \\
S_{t}\left(H_{k_{1}}\left(x_{j_{1}}\right) \cdot \ldots \cdot H_{k_{n}}\left(x_{j_{n}}\right)\right) & =z_{j_{1}}^{k_{1}} \cdot \ldots \cdot z_{j_{n}}^{k_{n}} \\
T\left(z_{j_{1}}^{k_{1}} \cdot \ldots \cdot z_{j_{n}}^{k_{n}}\right) & =x_{j_{1}}^{k_{1}} \otimes_{s} \ldots \otimes_{s} x_{j_{n}}^{k_{n}}
\end{aligned}
$$

The Segal-Bargmann map $S_{t}$ has been studied recently in different settings ([14], [15], [16]). We concentrate on the Taylor map $T$ since the Segal-Bargmann transform for a finite-dimensional Lie group $K_{\mathbb{C}}$ requires the inner product on the Lie algebra to be $A d_{K}$-invariant. The following diagram illustrates the maps described above including the ground state transformation, the Segal-Bargmann transform and the Taylor map.

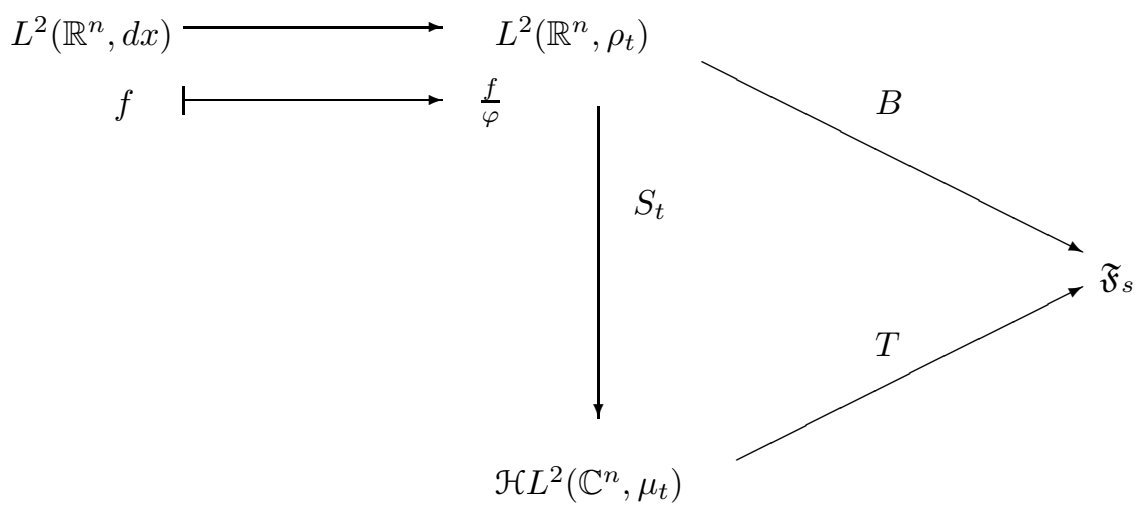

The Taylor map can be alternatively described as follows. First, we can identify the $k$ th derivative $D^{k}$ of a function $f$ with symmetric tensors by

$$
\left\langle D^{k} f(z), e_{i_{1}} \otimes \ldots \otimes e_{i_{k}}\right\rangle=\frac{\partial^{k} f}{\partial z_{i_{1}} \ldots \partial z_{i_{k}}}(z),
$$

where $\left\{e_{i}\right\}$ is an o.n. basis of $\mathbb{C}^{n}$. Then $T f=\sum_{k} D^{k} f(0)$ is in $\mathfrak{F}_{s}$, is surjective and satisfies the identity

$$
\|f\|_{\mathcal{H} L^{2}\left(\mathbb{C}^{n}, \mu_{t}\right)}^{2}=\int_{\mathbb{C}^{n}}|f(z)|^{2} d \mu_{t}(z)=\sum_{k=0}^{\infty} \frac{t^{k}}{k !}\left|D^{k} f(0)\right|^{2} .
$$

This identity is a commutative version of Equation 1.1. 
1.3. Infinite-dimensional commutative case. Let $\left(B, H_{C M}, \mu\right)$ be a complex Wiener space, where $B$ is a complex separable Banach space, $H_{C M}$ is the CameronMartin subspace (a complex separable Hilbert space which is densely and continuously embedded in $B$ ), and $\mu$ is a Gaussian measure defined by its characteristic functional

$$
\int_{B} e^{i \varphi(z)} d \mu(z)=e^{-\frac{1}{4}\|\varphi\|_{H_{C M}^{*}}^{2}, \varphi \in B^{*} \subset H_{C M}^{*} .}
$$

By using weak Cauchy-Riemann equations I.Shigekawa and H.Sugita in [19], [22], [23] introduced holomorphic functions on $(B, \mu)$. In this case the space of holomorphic $L^{2}(B, \mu)$ is a collection of $L^{2}(B, \mu)$-equivalency classes rather than functions as it was for finite-dimensional Lie groups. Still for each $f \in \mathcal{H} L^{2}(B, \mu)$ there exists a uniquely defined regular version (holomorphic skeleton) of $f$ on $H_{C M}$. The following pointwise estimate explains why the holomorphic skeleton is defined only on the Cameron-Martin subspace $H_{C M}$ which is of $\mu$-measure 0 .

$$
|f(h)|^{2} \leqslant\|f\|_{L^{2}(B, \mu)}^{2} e^{\frac{1}{2}\|h\|_{C M}^{2}}, h \in H_{C M} .
$$

We will have similar pointwise estimates for infinite-dimensional groups in Section 5.

1.4. New features in the infinite-dimensional noncommutative case. Let us list the problems that arise when we try to prove analogous results for infinitedimensional groups. First of all, we need a new construction to define a heat kernel measure. For the groups we consider there is no Haar measure. Moreover, the PDE theory is not developed enough to introduce a heat kernel as the fundamental solution to the heat equation. To deal with this problem we use the probabilistic approach described for finite-dimensional Lie groups earlier in this section and the theory of stochastic differential equations in infinite dimensions. This allows us to construct a heat kernel measure.

Another problem is how to define holomorphic functions. As we showed in [7], [8] and [9], there might be no nonconstant square integrable holomorphic functions. This question is related to another interesting problem which is new in infinite dimensions. We have a choice of natural Lie algebras, and this choice depends on the norm on this infinite-dimensional Lie algebra. By changing the norm, we change the Lie algebra as a set making it larger or smaller. At the same time, the Lie algebra determines the Laplacian, which in turn defines the heat kernel measure. Note that the Lie algebra is also the set of directions of differentiation for holomorphic functions. Thus if we choose a larger Lie algebra, the space of holomorphic functions becomes smaller, and in some cases the space of holomorphic square-integrable functions is trivial.

We also introduce an analogue of the Cameron-Martin subspace and holomorphic skeletons. The Cameron-Martin group can be described as the image of the infinitedimensional Lie algebra under the exponential map. This image has measure 0 , but this is the set where holomorphic skeletons live.

\section{Non-COMmutative $L^{p}$-SPaCes.}

Suppose $M \subseteq B(H)$ is a von Neumann algebra on a (separable) Hilbert space $H$, that is, $M$ is a $C^{*}$-algebra which is weakly closed and contains the identity operator $I$. Let $\tau$ be a faithful normal semifinite trace on $M$. This means that $\tau$ 
is a functional on the positive elements of $M$ satisfying the following properties for any $A, B \in M^{+}$

(1) $\tau(A+B)=\tau(A)+\tau(B)$,

(2) $\tau(c A)=c \tau(A)$ for any $0 \leqslant c \leqslant \infty, \tau(A B)=\tau(B A)$,

(3) $\tau$ is faithful means that $\tau(A)=0$ implies $A=0$,

(4) $\tau$ is normal means that $\tau\left(A_{\alpha}\right) \rightarrow \tau(A)$ for any increasing net $A_{\alpha}$ converging to $A$ in the strong operator topology,

(5) $\tau$ is semifinite means that $\tau(A)=\sup \left\{\tau(B): B \in M^{+}, B \leqslant A, \tau(B)<\infty\right\}$.

Example 2.1. $M=B(H), \tau(A)=\operatorname{Tr} A$ is the standard trace. This is a faithful normal semifinite trace.

Example 2.2. Abelian von Neumann algebra $M=L^{\infty}(X, \Omega, m)$, where $m$ is a probability measure, $H=L^{2}(X, \Omega, m)$. Let $\tau(f)=\int_{X} f d \nu$, where $\nu$ is a semifinite localizable measure on $(X, \Omega)$. Then $\tau$ is a faithful normal semifinite trace.

Example 2.3. A hyperfinite $\mathrm{II}_{1}$-factor (as the weak closure of a subalgebra of the CAR-algebra).

Definition 2.1. Noncommutative $L^{p}(M, \tau), 1 \leqslant p<\infty$ is a completion of the ideal $\{A \in M: \tau(|A|)<\infty\}$ in the norm

$$
\begin{aligned}
\|A\|_{p} & =\left(\tau\left(|A|^{p}\right)\right)^{1 / p}, 1 \leqslant p<\infty, \\
\|A\|_{\infty} & =\|A\|, \text { the operator norm. }
\end{aligned}
$$

The elements of $L^{p}(M)$ may be identified with (possibly unbounded) operators on $H$. It is well known that $\|\cdot\|_{p}$ is a norm and $L^{p}(M)$ is a normed vector space. This norm has the properties

$$
\left\|A^{*}\right\|_{p}=\||A|\|_{p}=\|A\|_{p},\|A B\|_{p} \leqslant\|A\|_{p}\|B\|, A \in L^{p}(M), B \in M .
$$

Example 2.4. $M=B(H), \tau(A)=\operatorname{Tr} A$. Then $L^{p}(M, \tau)$ is the $p$ th Schatten class, in particular, $L^{2}(M, \tau)$ is the space of Hilbert-Schmidt operators and $L^{1}(M, \tau)$ is the space of trace-class operators on $H$.

Example 2.5. Abelian von Neumann algebra $M=L^{\infty}(X, \Omega, m)$, where $m$ is a probability measure, $H=L^{2}(X, \Omega, m)$. Let $\tau(f)=\int_{X} f d \nu$, where $\nu$ is a semifinite localizable measure on $(X, \Omega)$. Then $L^{p}(M, \tau) \cong L^{p}(X, \Omega, \nu)$.

\section{Stochastic Differential equations on $L^{2}(M)$}

Let $Q$ be a bounded linear symmetric nonnegative operator on $L^{2}(M)$. Denote by $M_{Q}$ the subspace $Q^{1 / 2} L^{2}(M)$ with the inner product

$$
(A, B)=\left(Q^{-1} A, B\right)_{L^{2}(M)}
$$

and the corresponding norm by $|\cdot|$. Assume that $M_{Q} \subseteq M$. Denote by $W_{t}$ a Wiener process in $L^{2}(M)$ with the covariance operator $Q$, that is,

$$
W_{t}=\sum_{i=1}^{\infty} \frac{1}{\sqrt{2}} W_{t}^{i} \xi_{i},
$$


where $W_{t}^{i}$ are one-dimensional independent real Wiener processes and $\left\{\xi_{j}\right\}_{j=1}^{\infty}$ is an orthonormal basis of $M_{Q}$ as a real space.

Let $B, F: L^{2}(M, \tau) \rightarrow L^{2}(M, \tau)$. We assume that $\sum_{j=1}^{\infty} \xi_{j}^{*} \xi_{j}, \sum_{j=1}^{\infty} \xi_{j} \xi_{j}^{*}, \sum_{j=1}^{\infty} \xi_{j}^{2}$ are bounded operators with the series convergent in the operator norm.

Theorem 3.1 (SDEs [11]). Suppose that $Q$ is a trace-class operator. Then

(1) Suppose $F$ and $B$ are Lipschitz continuous on $L^{2}(M)$. Then the stochastic differential equation

$$
\begin{aligned}
& d X_{t}=F\left(X_{t}\right) d t+d W_{t} B\left(X_{t}\right), \\
& X_{0}=\xi, \xi \in L^{2}(M, \tau)
\end{aligned}
$$

has a unique solution in $L^{2}(M)$.

(2) The function $v(t, X)=P_{t} \varphi(X)$ is a unique solution for the parabolic type equation

$$
\frac{\partial}{\partial t} v(t, X)=\frac{1}{2} \sum_{j=1}^{\infty} v_{X X}(t, X)\left(\xi_{j} B(X) \otimes \xi_{j} B(X)\right)+\left(F(X), v_{X}(t, X)\right)_{L^{2}(M)} .
$$

Example 3.1. $M=B(H), \tau(A)=\operatorname{Tr} A$. Let $e_{i j}$ be the matrix with 1 at the $i j$ th place and 0 at all other places. Define $Q e_{i j}=\lambda_{i j} e_{i j}, \lambda_{i j} \geqslant 0$. Then $\xi_{i j}=\sqrt{\lambda_{i j}} e_{i j}$ is an orthonormal basis of $M_{Q}=Q^{1 / 2} L^{2}(M)$ and $W_{t}=\sum_{i, j} \frac{1}{\sqrt{2}} W_{t}^{i j} \xi_{i j}$, where $W_{t}^{i j}$ are one-dimensional independent real Wiener processes. In this case $Q$ is a trace class operator if and only if $\sum_{i, j} \lambda_{i j}<\infty$. Assume in addition that $\lambda_{i j}=\lambda_{i} \lambda_{j}$ (e.g. $\left.\lambda_{i j}=r^{i+j}, 0<r<1\right)$, then

$$
\sum_{i, j=1}^{\infty} \xi_{i j}^{*} \xi_{i j}=\sum_{i, j=1}^{\infty} \xi_{i j} \xi_{i j}^{*}=\sum_{i, j=1}^{\infty} \lambda_{i} \lambda_{j} e_{i i}, \sum_{i, j=1}^{\infty} \xi_{i j}^{2}=\sum_{i=1}^{\infty} \lambda_{i}^{2} e_{i i} .
$$

Example 3.2. Abelian von Neumann algebra $L^{2}(M, \tau)=L^{2}(0,1)$ with the Lebesgue measure. Then $e_{n}=\sqrt{2} \sin (n \pi x)$ is an orthonormal basis of $L^{2}(0,1)$. Suppose $Q$ is diagonal in this basis $Q e_{n}=\lambda_{n} e_{n}, \xi_{n}=\sqrt{\lambda_{n}} e_{n}$. Define $g(x)=\sum_{n=1}^{\infty} \xi_{n}^{*} \xi_{n}=$ $\sum_{n=1}^{\infty} \xi_{n} \xi_{n}^{*}=\sum_{n=1}^{\infty} \xi_{n}^{2}$. If $Q$ is a trace-class operator, then

$$
0 \leqslant g(x) \leqslant 2 \operatorname{tr} Q I \in B\left(L^{2}(0,1)\right) .
$$

In particular, if $Q^{-1}=-\Delta$, then

$$
g(x)=G(x, x),
$$

where $G(x, y)$ is Green's function for the Dirichlet problem on $[0,1]$.

\section{Heat kernel measures}

Let 


$$
B(X)=X, F(X)=\frac{1}{2} \sum_{j=1}^{\infty} \xi_{j}^{2} X .
$$

Then Kolmogorov's backward equation becomes the (group) heat equation.

Definition 4.1. The heat kernel measure $\mu_{t}$ on $L^{2}(M)$ is the transition probability of the stochastic process $X_{t}$.

The next theorem address the issue of whether the heat kernel measure actually lives in the group. This result shows that the process determining the measure has a one-sided inverse, which is a double-sided inverse for the case of Hilbert-Schmidt groups (see Section 6).

Theorem 4.2 (Inverse [11]). Denote

$$
T=\frac{1}{2} \sum_{j=1}^{\infty} \xi_{j}^{2}
$$

Then the solutions of the following stochastic differential equations

$$
\begin{aligned}
& d X_{t}=T X_{t} d t+d W_{t} X_{t}, \\
& d Z_{t}=Z_{t} T d t-Z_{t} d W_{t}, \\
& X_{0}=x, Z_{0}=z, \quad x, z \in L^{2}(M, \tau)
\end{aligned}
$$

satisfy

$$
Z_{t} X_{t}=z x
$$

with probability 1 for any $t>0$.

\section{Cameron-Martin group And isometries.}

The following definition was first given in [8].

Definition 5.1. The Cameron-Martin group is $G_{C M}=\left\{x \in M^{\times}, d(x, I)<\infty\right\}$, where $d$ is the Riemannian metric induced by $|\cdot|$ :

$$
d(x, y)=\inf _{\substack{g(0)=x \\ g(1)=y}}^{1}\left|g(s)^{-1} g^{\prime}(s)\right| d s
$$

Let $\mathcal{H} L^{2}\left(\mu_{t}\right)$ be the closure in $L^{2}\left(\mu_{t}\right)$ of holomorphic polynomials $\mathcal{H} \mathcal{P}$ on $L^{2}(M)$.

Theorem 5.2 (Holomorphic skeletons [8]). For any $f \in \mathcal{H} L^{2}\left(\mu_{t}\right)$ there is a holomorphic function $\tilde{f}$ on $G_{C M}$ such that for any $x \in G_{C M}$ and $p_{m} \in \mathcal{H} \mathcal{P}$

$$
\text { if } p_{m} \stackrel{L^{2}\left(\mu_{t}\right)}{\longrightarrow} f \text {, then } p_{m}(x) \longrightarrow \tilde{f}(x) \text {. }
$$

This skeleton $\tilde{f}$ is given by the formula

$$
\tilde{f}(x)=\sum_{k=0}^{\infty} \int_{0 \leqslant s_{1} \leqslant \ldots \leqslant s_{k} \leqslant 1}\left(D_{I}^{k} f\right)\left(c\left(s_{1}\right) \otimes \ldots \otimes c\left(s_{k}\right)\right) d \vec{s},
$$

where $D^{k} f$ is the $k$ th derivative of function $f$, and $c(s)=g(s)^{-1} g^{\prime}(s)$ for any smooth path $g(s)$ from I to $x$. 
The Cameron-Martin group can be also described by a certain finite-dimensional approximation. Let $M_{n}$ be finite dimensional von Neumann subalgebras, $G_{n}$ are the groups of invertible elements of $M_{n}$ with Lie algebras $M_{1} \subset M_{2} \subset \ldots \subset M_{n} \subset$ $\ldots \subset M_{Q}$. Assume that all $M_{n}$ are invariant subspaces of $Q$.

Notation 5.3. $G_{\infty}=\bigcup G_{n}$ and $\mathfrak{g}=\bigcup M_{n}$.

Remark 5.4. If $|[X, Y]| \leqslant c|X||Y|$, then $G_{\infty}$ is dense in $G_{C M}$ in the Riemannian metric induced by $|\cdot|$. This is the case for some Hilbert-Schmidt complex groups.

We also introduce one more (larger) space of holomorphic functions.

Definition 5.5. $\mathcal{H}^{t}\left(G_{C M}\right)$ is the space of holomorphic functions on the CameronMartin group $G_{C M}$ such that

$$
\|f\|_{t, \infty}=\lim _{n \rightarrow \infty}\|f\|_{t, n}=\lim _{n \rightarrow \infty} \int_{G_{n}}|f(z)|^{2} d \mu_{t}^{n}(z)<\infty .
$$

Theorem 5.6 (Pointwise estimates [7], [8], [9]). For any $f \in \mathcal{H}^{t}\left(G_{C M}\right), g \in G_{C M}$, $0<s<t$

$$
\left|\left(D^{k} f\right)(g)\right|_{\left(\mathfrak{g}^{*}\right) \otimes k}^{2} \leqslant \frac{k !}{s^{k}}\|f\|_{t, \infty}^{2} \exp \left(\frac{d^{2}(g, I)}{t-s}\right) .
$$

Theorem 5.7 (Isometries [7], [8], [9]). (1) The skeleton map is an isometry from $\mathcal{H} L^{2}\left(\mu_{t}\right)$ to $\mathcal{H}^{t}\left(G_{C M}\right)$, that is, the restriction map on holomorphic polynomials $\mathcal{H} \mathcal{P}$ extends to an isometry between the spaces $\mathcal{H} L^{2}\left(\mu_{t}\right)$ and $\mathcal{H}^{t}\left(G_{C M}\right)$.

(2) If $Q$ is a trace class operator, then $\mathcal{H} L^{2}\left(\mu_{t}\right)$ is an infinite-dimensional Hilbert space.

(3) The Taylor map $f \mapsto \sum_{k=0}^{\infty} D^{k} f(I)$ is an isometry from $\mathcal{H}^{t}\left(G_{C M}\right)$ to the generalized Fock space $\mathfrak{F}_{t}(\mathfrak{g})$, a subspace of the dual of the tensor algebra of $\mathfrak{g}$ with the norm

$$
|\alpha|_{t}^{2}=\sum_{k=0}^{\infty} \frac{t^{k}}{k !}\left|\alpha_{k}\right|^{2}
$$

Theorem 5.7 can be illustrated by the following diagram.

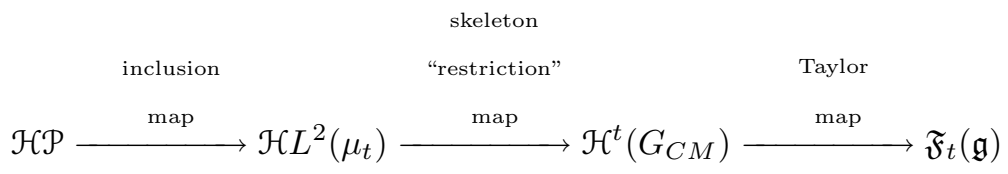

The generalized bosonic Fock space $\mathfrak{F}_{t}(\mathfrak{g})$ is a Hilbert space in the dual of the universal enveloping algebra of the Lie algebra $\mathfrak{g}$ defined as follows. Let $T(\mathfrak{g})$ be the tensor algebra over $\mathfrak{g}, J$ be the two-sided ideal in $T(\mathfrak{g})$ generated by $\{\xi \otimes \eta-\eta \otimes \xi-$ $[\xi, \eta] ; \xi, \eta \in \mathfrak{g}\}$. The algebraic dual of the tensor algebra $T(\mathfrak{g})$ is $T^{\prime}(\mathfrak{g})=\sum_{k=0}^{\infty}\left(\mathfrak{g}^{\otimes k}\right)^{*}$. Then $J^{0}$ is the annihilator of $J$ in the dual space $T^{\prime}(\mathfrak{g})$. Define 


$$
\|\alpha\|_{t}^{2}=\sum_{k=0}^{\infty}\left(\frac{t^{k}}{k !}\right)\left|\alpha_{k}\right|_{(\mathfrak{g} \otimes k}^{2}, \quad \alpha=\sum_{k=0}^{\infty} \alpha_{k}, \alpha_{k} \in\left(\mathfrak{g}^{\otimes k}\right)^{*}, k=0,1,2, \ldots
$$

Then the generalized bosonic Fock space is

$$
\mathfrak{F}_{t}(\mathfrak{g})=\left\{\alpha \in J^{0}:\|\alpha\|_{t}^{2}<\infty\right\} .
$$

This is the space of Taylor coefficients of functions from the space $\mathcal{H}^{t}\left(G_{C M}\right)$.

\section{EXAMPLES OF GROUPS}

In this section we describe examples of infinite-dimensional groups for which our construction works. Roughly speaking, we would like to consider $L^{2}(M, \tau)$ as the Lie algebra $\mathfrak{g}$, and the multiplicative group of $M$ as the infinite-dimensional (Lie) group. As we have seen $\mathfrak{g}$ actually has to be chosen smaller than the whole space $L^{2}(M, \tau)$ to ensure that the Brownian measure lives in the group.

6.1. Hilbert-Schmidt groups. Let $M=B(H)$ be the space of bounded linear operators on a complex Hilbert space $H$. Then the group is $M^{\times}=G L(H)$, invertible elements of $B(H)$. As before let $\tau(A)=\operatorname{Tr} A$ be the standard trace, then $L^{2}(M, \tau)=H S$ is the space of Hilbert-Schmidt operators on $H$ with the inner product $(\cdot, \cdot)_{H S}$. Suppose $\mathfrak{g} \subseteq H S$ is an infinite dimensional Lie algebra with a Hermitian inner product $(\cdot, \cdot)$ which in general is different from the Hilbert-Schmidt inner product.

Note that we can view elements of $H S$ as infinite matrices, namely, matrices $\left\{a_{i j}\right\}$ such that $\sum_{i, j}\left|a_{i j}\right|^{2}<\infty$. Let as before $e_{i j}$ be the matrix with 1 at the $i j$ th place and 0 at all other places. Assume that $Q e_{i j}=\lambda_{i j} e_{i j}, \lambda_{i j} \geqslant 0$. Then $\xi_{i j}=\sqrt{\lambda_{i j}} e_{i j}$ is an orthonormal basis of $M_{Q}$, and the Wiener process $W_{t}=\sum_{i, j} W_{t}^{i j} \xi_{i j}$. Note that $Q$ is a trace class operator if and only of $\sum_{i, j} \lambda_{i j}<\infty$.

Example 6.1. The Hilbert-Schmidt general group $G L_{H S}=G L(H) \cap(I+$ $H S$ ) with the Lie algebra $\mathfrak{g}=Q^{1 / 2} H S$ equipped with the norm $|A|=\left|Q^{-1 / 2} A\right|_{H S}$.

Example 6.2. The Hilbert-Schmidt orthogonal group $S O_{H S}$ is the connected component of $\left\{B: B-I \in H S, B^{T} B=B B^{T}=I\right\}$. Let $\mathfrak{s o}_{H S}=\{A: A \in$ $\left.H S, A^{T}=-A\right\}$, then the Lie algebra for $S O_{H S}$ is $\mathfrak{g}=Q^{1 / 2} \mathfrak{s o}_{H S}$ equipped with the norm $|A|=\left|Q^{-1 / 2} A\right|_{H S}$.

Example 6.3. The Hilbert-Schmidt symplectic group $S p_{H S}=\{X: X-I \in$ $\left.H S, X^{T} J X=J\right\}$, where $J=\left(\begin{array}{cc}0 & -I \\ I & 0\end{array}\right)$. Let $\mathfrak{s p}_{H S}=\left\{X: X \in H S, X^{T} J+J X=\right.$ $0\}$. Then the Lie algebra is $\mathfrak{g}=Q^{1 / 2} \mathfrak{s p}_{H S}$ with the norm $|A|=\left|Q^{-1 / 2} A\right|_{H S}$.

Suppose the operator $Q$ is as above.

Theorem 6.1. Let $Q: H S \rightarrow H S\left(\right.$ or $\mathfrak{s o}_{H S}$ or $\mathfrak{s p}_{H S}$ ).

(1) If $Q$ is trace class, then the heat kernel measure lives in $G L_{H S}$ (or $S O_{H S}$ or

$\left.S p_{H S}\right)$, and $\mathcal{H}^{t}\left(G_{C M}\right)$ is an infinite dimensional Hilbert space.

(2) If the covariance operator $Q$ is the identity operator, then $\mathcal{H}^{t}\left(G_{C M}\right)$ contains only constant functions. 
Remark 6.2. If the underlying Hilbert space $H$ is finite-dimensional, then these are just usual groups $G L, S O$ and $S p$. Thus the finite-dimensional groups can be viewed as a particular case of our setting.

Remark 6.3. It is known that the Riemannian geometry plays an important role in understanding of how the heat kernel measure behaves. It is a much more complicated issue in infinite dimensions. We address this issue in [12].

6.2. A hyperfinite $\mathbf{I I}_{1}$-factor (as the weak closure of a subalgebra of the CAR-algebra). In the following description we follow L. Gross [13] and I. E. Segal [20]. Let $K$ be a complex Hilbert space with a Hermitian inner product $(\cdot, \cdot)_{K}$. Denote by $\Lambda^{n}(K)$ the space of skew symmetric tensors of rank $n$ over $K$, and $\Lambda^{0}(K)=\mathbb{C}, H=\Lambda(K)=\oplus_{n=0}^{\infty} \Lambda^{n}(K)$. For any $x$ in $K$ there exists a bounded creation operator $C_{x}$ on $H=\Lambda(K)$ such that

$$
C_{x} u=(n+1)^{\frac{1}{2}} x \wedge u, u \in \Lambda^{n}(K),
$$

where $x \wedge u$ is the antisymmetric projection of $x \otimes u$.

Then the annihilation operator is $A_{x}=C_{x}^{*}$ and

$$
C_{x} A_{y}+A_{y} C_{x}=(x, y)_{K} I .
$$

Fix a conjugation $\mathcal{f}$ on $K$ and define

$$
B_{x} \stackrel{\text { def }}{=} C_{x}+A_{\mathcal{\partial} x}
$$

Then

$$
B_{x} B_{y}+B_{y} B_{x}=2(x, J y)_{K} I .
$$

The von Neumann algebra $M$ is the smallest weakly closed algebra of operators on $\Lambda(K)$ containing all the operators $B_{x}, x \in K$, that is, $M \subseteq B(\Lambda(K))=B(H)$. The trace is defined by $\tau(u)=(u \Omega, \Omega)$, where the "bare" vacuum $\Omega=1$ is an element in $\Lambda^{0}(K) \subset \Lambda(K)$. It is known that the space $M$ with this trace is a hyperfinite $\mathrm{II}_{1}$-factor [20]. As always, the trace determines a Hermitian inner product on $M$ by $(A, B)_{L^{2}(M)}=\tau\left(B^{*} A\right)$ and $L^{p}(M)$ is the completion of $M$ in the norm $\|A\|_{p}=\left(\tau\left(|A|^{p}\right)\right)^{\frac{1}{p}}$ for $1 \leqslant p<\infty$.

One of the advantages of this representation of a hyperfinite $\mathrm{II}_{1}$-factor is that we can use a natural orthonormal basis of $L^{2}(M)$ as follows. It is known that if $\left\{x_{i}\right\}_{1}^{\infty}$ is an orthonormal basis of $K$, then $B_{x_{i_{1}}} \ldots B_{x_{i_{n}}}$ is an orthonormal basis of $L^{2}(M)$ $\left(i_{1}<\ldots<i_{n}, n \geqslant 0\right)$.

Let $Q: L^{2}(M) \rightarrow L^{2}(M), \mathfrak{g}=Q^{1 / 2} L^{2}(M)$.

Remark 6.4. If $Q$ is a trace class operator, then the results from Section 5 (the skeletons, pointwise estimates and isometries) hold.

Theorem 6.5 ([9]). If $Q$ is the identity operator, then $\mathcal{H}^{t}\left(G_{C M}\right)$ contains only functions of the form

$$
f(X)=g\left((X, I)_{L^{2}(M)}\right),
$$

where $g(\cdot)$ is a holomorphic function of one complex variable. 


\section{REFERENCES}

[1] V.Bargmann, On a Hilbert space of analytic functions and an associated integral transform, Part I, Communications of Pure and Applied Mathematics, 24, 1961, 187-214.

[2] V.Bargmann, Remarks on a Hilbert space of analytic functions, Proc. of the National Academy of Sciences, 48, 1962, 199-204.

[3] G.DaPrato and J.Zabczyk, Stochastic Equations in Infinite Dimensions, Cambridge University Press,Cambridge, 1992.

[4] B.Driver, On the Kakutani-Itô-Segal-Gross and Segal-Bargmann-Hall isomorphisms, J. of Funct. Anal., 133,1995,69-128.

[5] B.Driver and L.Gross, Hilbert spaces of holomorphic functions on complex Lie groups, Proceedings of the 1994 Taniguchi International Workshop (K. D. Elworthy, S. Kusuoka, I. Shigekawa, Eds.), World Scientific Publishing Co. Pte. Ltd, Singapore/New Jersey/London/Hong Kong, 1997.

[6] E. B. Dynkin, Calculation of the coefficients in the Campbell-Hausdorff formula, Doklady Akad. Nauk SSSR (N.S.) 57, 1947, 323-326.

[7] M. Gordina, Holomorphic functions and the heat kernel measure on an infinite-dimensional complex orthogonal group, Potential Analysis Volume, 12, 2000, pp. 325-357.

[8] M. Gordina, Heat kernel analysis and Cameron-Martin subgroup for infinite-dimensional groups, J. Func. Anal., 171, 2000, pp. 192-232.

[9] M. Gordina, Taylor map on groups associated with a II $I_{1}$-factor, 2002, Infin. Dimens. Anal. Quantum Probab. Relat. Top., 5, 93-111.

[10] M. Gordina, Quasi-invariance for the pinned Brownian motion on a Lie group, 2002, to appear in Stochastic Processes and Their Applications.

[11] M. Gordina, Stochastic differential equations on noncommutative $L^{2}, 2002$, to appear in Contemp. Math. book series of the Amer. Math. Soc. (proceedings of the Conference on Analysis on Infinite-Dimensional Spaces in honor of Leonard Gross), H-H. Kuo and A.N. Sengupta, Eds.

[12] M. Gordina, Hilbert-Schmidt groups: Riemannian geometry, Lie algebras etc., 2004, preprint.

[13] L. Gross, Existence and uniqueness of physical ground states, J. Funct. Anal., 10, 1972, 52-109.

[14] L. Gross and P. Malliavin, Hall's transform and the Segal-Bargmann map, Itô's Stochastic Calculus and Probability Theory, (M.Fukushima, N. Ikeda, H. Kunita and S. Watanabe, Eds.), Springer-Verlag, New York/Berlin, 1996.

[15] B.Hall, Quantum Mechanics in Phase Space, Proc. of the Summer Research Conf. on Quantization, (L. Coburn and M. Rieffel, Eds.), 1997.

[16] B.Hall, The Segal-Bargmann 'coherent state' transform for compact Lie groups, J. of Funct. Anal., 122, 1994, 103-151.

[17] B. Hall and A. Sengupta, The Segal-Bargmann transform for path-groups, J. of Funct. Anal., 152, 1998, 220-254.

[18] Robert H. Martin, Jr., Nonlinear operators and differential equations in Banach spaces, Pure and Applied Mathematics. Wiley-Interscience [John Wiley \& Sons], New York-LondonSydney, 1976.

[19] I. Shigekawa, Itô-Wiener expansions of holomorphic functions on the complex Wiener space, in Stochastic Analysis, (E. Mayer et al, Eds.), Academic Press, San Diego, 1991, 459-473.

[20] I. E. Segal, Tensor algebras over Hilbert spaces. II, Ann. of Math., 63, 1956, 160-175.

[21] I. E. Segal, Mathematical problems of relativistic physics, Lectures in Applied Mathematics (proceedings of the Summer Seminar, Boulder, Colorado, 1960), Vol. II American Mathematical Society, Providence, R.I.

[22] H.Sugita, Properties of holomorphic Wiener functions-skeleton, contraction, and local Taylor expansion, Probab. Theory Related Fields, 100, 1994, 117-130.

[23] H.Sugita, Regular version of holomorphic Wiener function, J. Math. Kyoto Univ., 34, 1994, 849-857.

Department of Mathematics, University of Connecticut, Storrs, CT 06269, U.S.A.

E-mail address: gordina@math.uconn.edu 